\title{
Desarrollo de el Parque Industrial El Salto, Jalisco*
}

\author{
Juan Jorge Rodríguez Bautista y \\ María del Rosario Cota Yánez**
}

Resumen: El Salto fue un municipio pionero en proyectos industriales en el país. Sus inicios como espacio manufacturero se remontan a 1866 cuando se instaló una planta procesadora de hilados, la cual aprovechó una caída de agua como fuente de energía. Posteriormente, en la década de los 60's se crea el corredor industrial El Salto donde se asentaron empresas del ramo electrónico de importancia mundial como son: IBM, Hitachi. A pesar de lo anterior, presenta una incorporación tardía al desarrollo económico regional y algunas de las empresas actualmente están trasladando sus plantas productivas a países asiáticos.

Palabras-clave: Parque Industrial El Salto, Industrialización, Empresas.

Abstract: El Salto was one of the first municipalities in Mexico to welcome industrial projects. Its history as a place for manufacturing started in 1866 with the installation of a mill for spinning thread, that used a waterfall for energy. A century later, in the nineteen sixties, when the industrial corridor of El Salto came into being, electronics companies of international standing such as IBM and Hitachi moved in. However, it took a long time for El Salto to be incorporated into the economic development of the region, and now some of the companies working there are moving their productive plants out to Asian countries.

Keywords: Industrial Park, El Salto, Industrialization, Companies.

\footnotetext{
* Este documento fue presentado en el 52 Congreso Internacional de Americanistas, en Sevilla España. Julio del 2006. ** Juan Jorge Rodríguez Bautista: Profesor investigador del Departamento de Estudios Regionales del CUCEA-UDG y jefe del Departamento de Ciencias Económicas y Administrativas del CUVALLES. Miembro de la Benemérita Sociedad de Geografía del Estado de Jalisco y del Sistema Nacional de Investigadores. Pertenece al cuerpo académico Desarrollo Regional e Integración Económica. UDG-CA-118. E-mail: rbj14451@cucea.udg.mx. María del Rosario Cota Yánez: Profesora investigadora del Departamento de Estudios Regionales del CUCEA-UDG. Miembro del Sistema Nacional de Investigadores. Pertenece al cuerpo académico Desarrollo Regional e Integración Económica. UDG-CA-118. E-mail: macotaya@cucea.udg.mx. Recebido em 06/10/06 e aceito em 03/11/06.
} 


\section{Introducción}

El municipio de El Salto forma parte de la Zona Metropolitana de Guadalajara, en el estado de Jalisco, México. Se ubica al suroeste de la ciudad, cubre una superficie de 41 kilómetros cuadrados y cuenta con una población de 111436 habitantes para el 2005 de acuerdo al último Censo de Población y Vivienda (INEGI, 2000).

La participación de este municipio en la Zona Metropolitana de Guadalajara ha sido significativa en el desarrollo industrial. Desde el siglo XIX este territorio tuvo recursos hidrológicos que fueron aprovechados por empresas manufactures que sobresalieron en la región. Actualmente esos recursos sufren contaminación ${ }^{1}$.

Sin embargo, para mediados del siglo XX su desarrollo industrial se estancó, privilegiándose al municipio de Guadalajara, como el centro económico e industrial del estado y de la región occidente del país. Para ese entonces El Salto era un municipio rural ubicado a 15 kilómetros de la ciudad, por lo que difícilmente se podría pensar en una opción para que se localizaran las empresas manufactureras, debido a la falta de infraestructura y comunicación.

A finales de la década de los 60's, El Salto volvió aparecer como un espacio idóneo para el desarrollo industrial, ya que dentro de la política de desconcentración industrial del gobierno federal construyó el Parque Industria Guadalajara, en el cual se instalaron varias empresas importantes. Sin embargo, para esas fechas el modelo de industrialización estaba cambiando para favorecer a las zonas que contaran con infraestructura especialidad, mano de obra calificada y servicios avanzados. Guadalajara empezó ha desarrollar estas características, pero aisló completamente al municipio de El Salto, no se invirtió en infraestructura para atraer empresas manufactureras del sector moderno.

Así como se presenta el desequilibrio intrametropolitano, donde la inversión se concentra sólo en algunos municipios de las metrópolis. Es por

\footnotetext{
${ }^{1}$ Actualmente en los municipios de Juanacatlán y El Salto, Jalisco, la contaminación del Río Santiago está generando un problema de salud pública alarmante. El Río Santiago es uno de los más contaminados del país y las aguas que caen por la cascada denominada el Salto de Juanacatlán son fuente de gases y olores fétidos que se estudian y comprueban como causas de enfermedad y muerte. El programa de saneamiento propuesto por el estado de Jalisco es deficiente (Instituto Mexicano para el Desarrollo Comunitario AC, 2007).
} 
ello que se muestran grandes avances en ciertas zonas de la ciudad y otras quedaron como espectadores del desarrollo industrial y urbano.

Durante el último proceso de industrialización en la zona Metropolitana de Guadalajara, El Salto, tuvo la función de recibir algunas empresas; sin embargo no ha sido suficiente para desenvolverse como un municipio metropolitano exitoso, por el contrario, esto le ha atraído serios problemas en la dotación de servicios públicos y sociales hacia la población.

En ese sentido, se sostiene que El Salto juega un papel dentro de la metrópoli como el lugar donde se asienta la población segregada del desarrollo urbano, ya que ahí no se ha dotado de la infraestructura suficiente para satisfacer las necesidades de la población.

Para comprobar lo anterior, este artículo analiza, en una primera parte, cómo las ciudades se han transformado en las diferentes etapas de la industrialización. En México, el modelo de sustitución de importaciones generó un binomio: urbanización-industrialización. En cambio para el nuevo modelo de industrialización, donde el proceso de globalización condicionó las formas de producción y comercialización; se generó una concentración de algunas actividades, provocando funciones diferenciadas en cada una de las zonas de la ciudad.

En el segundo apartado se revisa la evolución industrial de El Salto, el cual se inicia desde finales del siglo XIX y se detecta como a lo largo del siglo XX su participación fue tardía, por lo que los efectos no fueron los más adecuados para alcanzar un desarrollo económico y social del municipio.

En el siguiente apartado se describe la función de El Salto, bajo las nuevas tendencias metropolitanas, para ese momento, y a pesar de localizarse una de las empresas más importes del ramo electrónico, no pudo insertarse al desarrollo de este ramo manufacturero, dejando a los municipios de Zapopan, Tlajomulco y Tlaquepaque como los espacios donde de implantaron la mayor parte de las empresas.

Finalmente se plantean algunas reflexiones tratando de identificar las razones por las cuales el municipio de El salto no pudo insertarse mejor en el proceso de desarrollo industrial de los últimos años y emprendió la función de ubicar a la población con escasos recursos. 


\section{Transformación de las metrópolis en las diferentes trayectorias industriales}

Las ciudades han estado en constantes cambios, debido a sus alteraciones en su función principal. Sin embargo, durante el siglo XX se detectó plenamente una única función, aglomerar población y actividades que permitían acceder a mejores condiciones de vida e impulsan el desarrollo económico de una región o nación.

Lo anterior generó una expansión urbana cada vez más compleja: urbanización, conurbación, metropolización, megalopolización y metapolización; provocando que dentro de las ciencias sociales se visualizara con mayor detenimiento el comportamiento económico, demográfico y social de las ciudades.

Esta tendencia de crecimiento físico de las ciudades buscó detenerse, reubicando población y actividades económicas en otros espacios, pretendiendo dar solución a los desequilibrios regionales, los cuales se manifestaban comúnmente en países y regiones poco desarrollados. Lo anterior generó nuevas aglomeraciones urbanas que alcanzaron en las siguientes décadas los mismos problemas de concentración poblacional y económica, manifestado años atrás por las grandes metrópolis.

Las ciudades emprendieron el camino de la industrialización, aprovechando la concentración de mano de obra, mercado e infraestructura necesaria para el desarrollo de esta actividad. En México este proceso se inicio en los años de 1940, cuando el modelo de sustitución de importaciones fue la política gubernamental que cobijó el desarrollo industrial en las ciudades. A la vez, surgieron otras políticas de desconcentración industrial que buscaron generar polos de crecimiento para equilibrar la dinámica económica del país.

Este proceso de industrialización estaba caracterizado por la producción en masas o fordista, el cual consistía en aprovechar las economías de escala con el objeto de producir grandes cantidades para aminorar costos. Esto generó grandes plantas industriales, lo que determinó el uso de maquinaria pesada y la incorporación de abundante mano de obra, propiciando una concentración urbana.

En las últimas tres décadas del siglo $\mathrm{XX}$, las ciudades tuvieron una transformación, conformando otro escenario económico y territorial. Esto 
tiene que ver con las tendencias de reestructuración productiva y las nuevas formas de organización espacial de las empresas.

Caravaca y Méndez (2003), coinciden en decir que la reestructuración productiva arrojó ciertos efectos como la pérdida de empleos y la ruptura en el proceso de crecimiento acumulativo que las aglomeraciones urbanas habían experimentado desde el inicio de la industrialización. Lo anterior llevó a pensar que se estaba ante el fin de la industrialización urbana, debido al surgimiento de otros sectores económicos y la desconcentración de las manufacturas.

Así aparecen nuevos conceptos como desindustrialización, postindustrialización, desurbanización o contraurbanización; poniendo en evidencia la crisis industrial metropolitana. Por lo que la nueva función de la ciudad sería la generación de servicios basados en la satisfacción de las necesidades sociales. Surge la tesis de la terciarización de las ciudades, provocada por la salida de las manufacturas de las áreas urbanas, ${ }^{2}$ y el desenvolvimiento de actividades que ofrecieran servicios a la población, debido a las nuevas formas de vida.

Sin embargo, existe otra tesis denominada Neoindustrial (CARAVACA, MÉNDEZ, 2003), que cuestiona lo anterior. Las ciudades se desplazan hacia los servicios debido a las nuevas formas de organización productiva, basada en las cadenas de valor mediante el aumento de la importancia de tareas previas (gestión, planificación, investigación, desarrollo y diseño) y posteriores a la fabricación (logística y almacenamiento, control de calidad, comercialización y servicios postventa).

En el ámbito territorial la industria ha distribuido funciones hacia diferentes espacios, propiciando una nueva división espacial del trabajo. Lo anterior genera dos efectos que se plantean en el nuevo funcionamiento de las ciudades: la dispersión de procesos productivos en espacios urbanos, semiurbanos y rurales; y la concentración de funciones que controlan los procesos productivos dispersos en las metrópolis.

Cada una de las ciudades empiezan a diferenciarse con base en su función dentro del proceso productivo; por un lado existen aquellas donde

\footnotetext{
${ }^{2}$ La desindustrialización de las ciudades fue provocada por las deseconomías de escala, las cuales se daban por la saturación de infraestructura, la escasez o encarecimiento del suelo y las recientes restricciones medioambientales (DE MATTOS, 2001).
} 
la industria es la base económica, generando infraestructura urbana y otras actividades para su desenvolvimiento. En cambio otras ciudades transforman su espacio urbano formando adecuados sistemas de comunicación y transporte, desarrollo de centros de investigación, incorporación de servicios especializados y recursos humanos cualificados.

El análisis territorial de la reestructuración productiva se enfoca también dentro de una escala intrametropolitana. Debido a su crecimiento desmedido y su amplia estructura poblacional y económica, los espacios urbanos pasan de una ciudad nuclear a una región metropolitana. Hoy en día, se está produciendo una estructura territorial compleja donde la interconexión entre las áreas de la metrópoli son cada vez más estrechas, aunque más distantes. Cada una de las áreas tiene sus funciones específicas que reafirman su incorporación al desarrollo económico de la ciudad o son los espacios segregados aunque necesarios para su función principal.

En el interior de la ciudad también existe desconcentración, la zona central reduce su participación industrial, facilitando su incorporación a la periferia urbana, conformándose una región metropolitana.

La evolución que han presentado las áreas urbanas a lo largo del siglo XX y los primeros años de éste, se ve reflejada de manera diferente, mientras que unas están incursionando hacia nuevas formas de industrialización, otras están en etapas postindustriales o neoindustriales con un predominio de los servicios avanzados dentro de la dinámica económica de la ciudad. Esto refuerza la posición funcional dominante de un espacio urbano en la nueva división espacial del trabajo, pero a costa de empresas, sectores y personas que tienden a ser expulsadas del tejido urbano hacia espacios periféricos.

En Guadalajara el proceso industrial no ha sido diferente, pero sí para cada uno de los municipios que conforman la zona metropolitana, particularmente El Salto, ya que a pesar de su inicio incipiente en el desarrollo industrial, este no se vinculó al desarrollo industrializador de la segunda parte del siglo XX, sino hasta que este proceso estuvo a punto de sufrir una transformación significativa, la cual dificultó su incorporación plena al nuevo esquema de industrialización vivida en la ciudad, por lo que sólo tuvo la función de incorporar población de bajos ingresos y segregada de los beneficios de las nuevas tendencias de desarrollo industrial y urbano. 


\section{Antecedente del proceso de industrialización de El Salto}

El Salto nace como municipio en 1943, por lo que es relativamente nuevo. Sin embargo, sus inicios como espacio industrial se remontan a 1866 cuando se instaló una planta manufacturera de hilados, la cual aprovechó una caída de agua como fuente de energía. Este fenómeno natural fue conocido como El Salto de Juanacatlán.

Para esos tiempos el modelo de industrialización, que se desarrolló en Inglaterra, consistía en instalar las plantas productivas fuera de las ciudades donde se tuvieran grandes espacios y dotar de todas las necesidades de los trabajadores (vivienda, diversión, consumo, entre otros), esto era construir una ciudad que fuera controlada por la fábrica (DURAND, 1992). Es así como El Salto inicia su etapa de industrialización

Para 1876 se instala otra fábrica de hilados denominada "Río Grande", la cual buscó aprovechar la misma fuente de energía. Dicha empresa se ubicó en los terrenos de la Hacienda Jesús María, cercana a la estación ferroviaria de El Castillo; y para llevar el mismo modelo de las áreas industriales europeas se creó la colonia Industrial de El Salto. Ahí llegaron a vivir cerca de mil trabajadores que venían del centro del país. Para finales del siglo XIX, la fábrica Río Grande se convirtió en la empresa textil más importante del Estado; sin embargo, el modelo de organización empezó a tener problemas debido al contexto político y la efervescencia de una lucha por la libertad por parte de obreros y campesinos (DURAND, 1992).

Otro acontecimiento que impactó esa zona de Jalisco al desarrollo industrial de finales de siglo XIX, fue la instalación del molino de harina y la Hidroeléctrica, instalados en 1880 y 1892 respectivamente. Esta última permitió generar un avance importante en la modernización de Guadalajara, ya que permitió abastecer de energía suficiente para iluminar las calles del primer cuadro de la ciudad y posteriormente toda la capital del Estado (DURAND, 1992).

Por lo anterior puede afirmarse que El Salto tuvo un inicio pujantemente en el desarrollo industrial de Jalisco, aprovechando las condiciones hidrológicas existentes, el espacio suficiente que había para desarrollar zonas industriales donde se conformaron poblados y el aprovechamiento de la infraestructura del ferrocarril que facilitó el acceso a otras zonas del país, aunque también 
influyó en la presencia de nuevos capitales nacionales y extranjeros que llegaron con otras estrategias industriales y comerciales, con el fin de acaparar el desarrollo agropecuario existente en la región.

Para la primera mitad del siglo XX, el desarrollo industrial se concentró en la capital del país. El modelo de sustitución de importaciones, iniciado en 1940, propició la concentración industrial sólo en los lugares donde se tenía la infraestructura adecuada y mano de obra abundante. Es así como, a nivel nacional las ciudades de México, Monterrey y Guadalajara fueron las que acapararon el desarrollo de este modelo económico productivo. Ello provocó una fuerte concentración económica y poblacional, ya que las empresas buscaban aprovechar las economías de escala que se ofrecían y la población se daba cuenta que en estos lugares podrían acceder a un empleo mejor remunerado y a servicios básicos y sociales que no podría encontrar en sus lugares de origen, esto hizo que se manifestaran en estos espacios urbanos mejores condiciones de vida.

La concentración económica y demográfica, llevó al país a un desequilibrio regional. Las ciudades crecían económicamente, mientras que el resto se quedaba estancado. El gobierno federal planteó una serie de estrategias las cuales pretendían eliminar o aminorar estas desigualdades.

Para 1953 surge el programa de Parques y Ciudades Industriales, que inició con la construcción de Ciudad Sahagún, un complejo industrial ubicado en el estado de Hidalgo, que pretendía desconcentrar las plantas manufactureras localizadas en la capital del país, a pesar de estar relativamente cerca. Durante este programa se crearon casi cien parques y ciudades industriales, cuatro de ellos en la Zona Metropolitana de Guadalajara. El primero en instalarse fue el Parque Industrial Guadalajara, ubicado en el municipio de El Salto en 1967, el objetivo de este parque era desconcentrar fábricas ubicadas en las zonas metropolitanas del país, principalmente de la ciudad de México y Guadalajara, considerando que El Salto podría ser una opción por el antecedente industrial obtenido décadas atrás y porque podría aprovechar las ventajas de aglomeración al estar cerca de Guadalajara.

Para ese tiempo se instalaron varias empresas relevantes que impactaron no sólo el desarrollo industrial del municipio, sino que empezó a tener una relación funcional más directa con la capital de Jalisco: Celulosa y Derivados 
(química y textil), Polisac (plásticos), Aceros Industrial (Metal mecánica), Champiñones de Guadalajara (alimenticia), Industrias Petroquímicas (Petroquímica), Euzkadi ${ }^{3}$ (Llantas), Maquiladora de Oleaginosas (aceitera), entre otras.

Para 1970, cuando la política sustitutiva de importaciones empezó a tener signos de agotamiento, los últimos esfuerzos por seguir el modelo de producción fordista vigente y la desconcentración industrial fue la instalación de la empresa electrónica IBM, que para ese entonces sólo producía máquinas de escribir y suministros. La situación vivida por El Salto en esta etapa permite afirmar que tuvo una incorporación tardía, a pesar de ser uno de los iniciadores de proyectos industriales importantes desde finales del siglo XIX en el Estado. El municipio de Guadalajara acaparó todo el proceso de industrialización debido a las necesidades de las empresas por aprovechar las economías de escala y el vínculo que se gestó entre el desarrollo industrial y urbano.

Laley de fomento industrial impulsada en desdela década de 1940 benefició la instalación de nuevas empresas en la ciudad, las cuales se concentraron en los municipios de Guadalajara, Zapopan y Tlaquepaque (ARIAS, 1992), ello limitó el desarrollo industrial de el Salto en prácticamente seis décadas.

La creación de zonas industriales se enfocó sólo al municipio de Guadalajara ya que concibió como un espacio ideal para la creación de esta infraestructura, es así como en 1950 aparece la Zona Industrial Guadalajara, ubicada a un lado de la estación del ferrocarril, con el objeto de aprovechar este medio de transporte para acercar materias primas de los centros agropecuarios de la región, así como trasladar insumos o productos finales hacia los diferentes mercados del país.

Otros espacios industriales desarrollados fueron el corredor industrial González Gallo y la zona industrial El Álamo, que se crearon en la década de 1960, en los límites con el municipio de Tlaquepaque. Estas zonas industriales permitieron fijar al municipio de El Salto como una opción para establecer el

\footnotetext{
${ }^{3}$ La empresa Euzkadi es de origen alemán y se dedica a la fabricación de neumáticos. Abrió sus puertas en 1971 y cesó operaciones el 16 de diciembre de 2001. Se liquidaron a 1164 trabajadores. Debido, según sus directivos "a varios intentos reiterados de aplicar estándares internacionales de productividad que no se ajustaron”. Manufacturaba 3 millones de llantas para automóvil y camión al año. Otra planta de Continental en Norteamérica suplirá esta producción (El Informador. Hemeroteca, 18 diciembre 2001).
} 
primer parque industrial del programa desarrollado por el gobierno federal en Jalisco, para desconcentrar la industria de las metrópolis.

Sin embargo, para mediados de la década de 1970, cuando se empezaron a localizar empresas manufactureras en El Salto y la reubicación de la planta industrial IBM, el modelo económico productivo generado desde la posguerra empezó a ser obsoleto en los países industrializados y en vías de industrialización, impactando fuertemente en países latinoamericanos como Brasil, Argentina, Colombia, entre otros; esto provocó dar paso a un nuevo paradigma: producción flexible y la apertura económica.

Dentro de este nuevo modelo de industrialización El Salto pudo haber participado estratégicamente; sin embargo, su adecuación a las nuevas condiciones que exigían las empresas trasnacionales de la industria electrónica, se desarrollaron más en el municipio de Zapopan, Tlaquepaque y Tlajomulco. Una de estas necesidades fue la creación de nuevos parques tecnológicos con infraestructura especializada, que se localizaron en zonas estratégicas de la ciudad para aprovechar las instituciones educativas y los servicios avanzados que se desarrollaron en el municipio de Guadalajara (Mapa 1). Actualmente se observa en El Salto un proceso de cierre de empresas del ramo electrónico importantes a nivel mundial como es el caso de Hitachi, ${ }^{4}$ que recientemente anunció su retiro de la localidad dejando desempleados a 4500 trabajadores.

De los 20 parques industriales existentes en la Zona Metropolitana de Guadalajara, sólo dos se ubican en El Salto, el último se instaló a finales de la década de 1980, el resto se instalaron en los municipios metropolitanos de Tlaquepaque, Zapopan y Tlajomulco, donde se tenia acceso a los mercados regionales más importantes del país (centro y norte) y las carreteras que

\footnotetext{
${ }^{4}$ Hitachi, empresa de origen japonés dedicado a la fabricación de artículos electrónicos. Será cerrada definitivamente a mediados del año próximo debido a "un proceso de reestructuración mundial en su sector de las unidades de disco duro" en palabras de sus directivos (El Informador, 22 de marzo de 2007). Su producción de componentes (sliders) de disco duro será trasladada a Filipinas. Con esta medida Hitachi se ahorrará 300 millones de dólares en cinco años. Ya que las tendencias en la producción de disco duro se concentran en toda Asia y Filipinas es un sitio cercano a sus plantas de montaje final en Tailandia y China. Hitachi en el año 2006 fue considerada una empresa socialmente responsable por adherir a su planta laboral a quienes regularmente pertenecen a grupos discriminados de una población como son mujeres, minusválidos y personas con Virus de Inmuno Deficiencia Adquirida. Como parte de la Nueva Cultura Laboral. Se debe de contratar como mínimo seis meses, la planta debe hacer adecuaciones de accesibilidad, traductores de lenguaje para la capacitación y sin hacer pruebas de VIH al nuevo personal.
} 
comunicaban a puertos y aeropuertos que trasladarían los productos a otras latitudes del mundo.

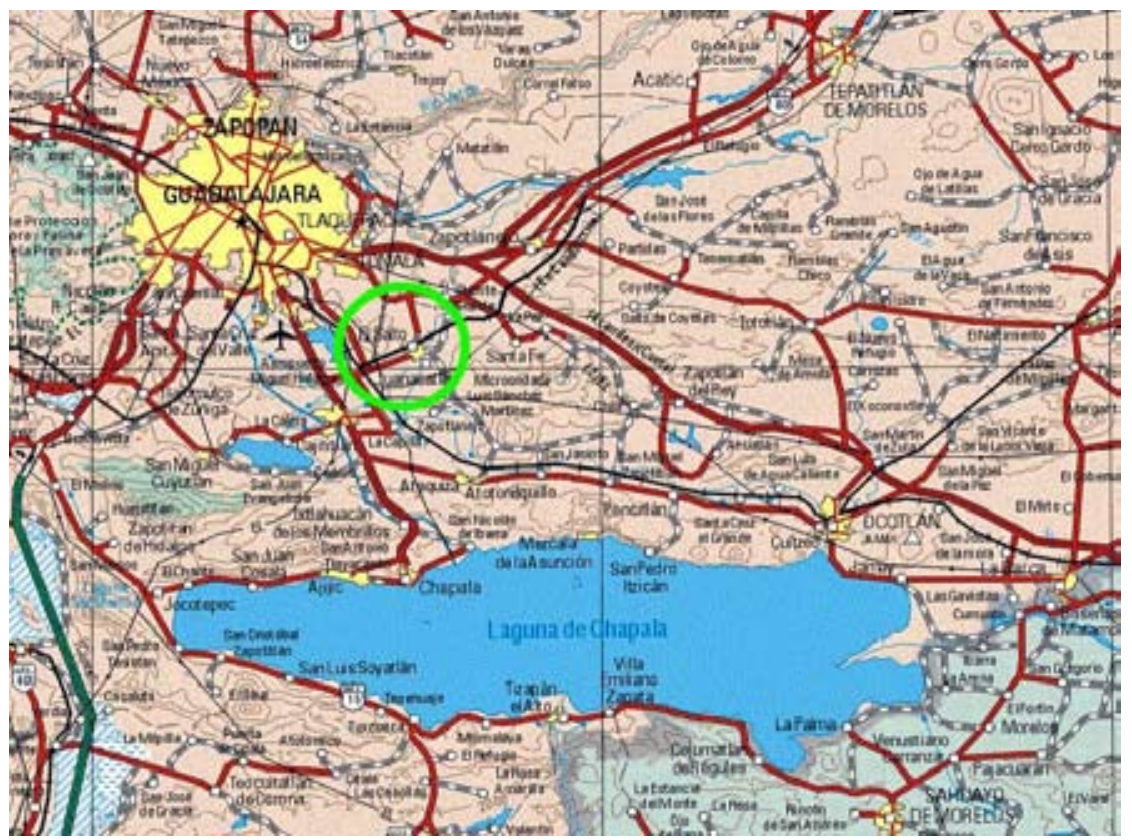

Mapa 1 - Ubicación del municipio de El Salto

La situación metropolitana de El Salto estuvo de la mano con el desarrollo industrial de la década de 1960, donde las primeras empresas instaladas provocaron la movilidad de mano de obra de la zona metropolitana a este municipio (Foto 1), pero no fue hasta la década de 1980, donde el municipio fue catalogado como parte de la zona metropolitana. 


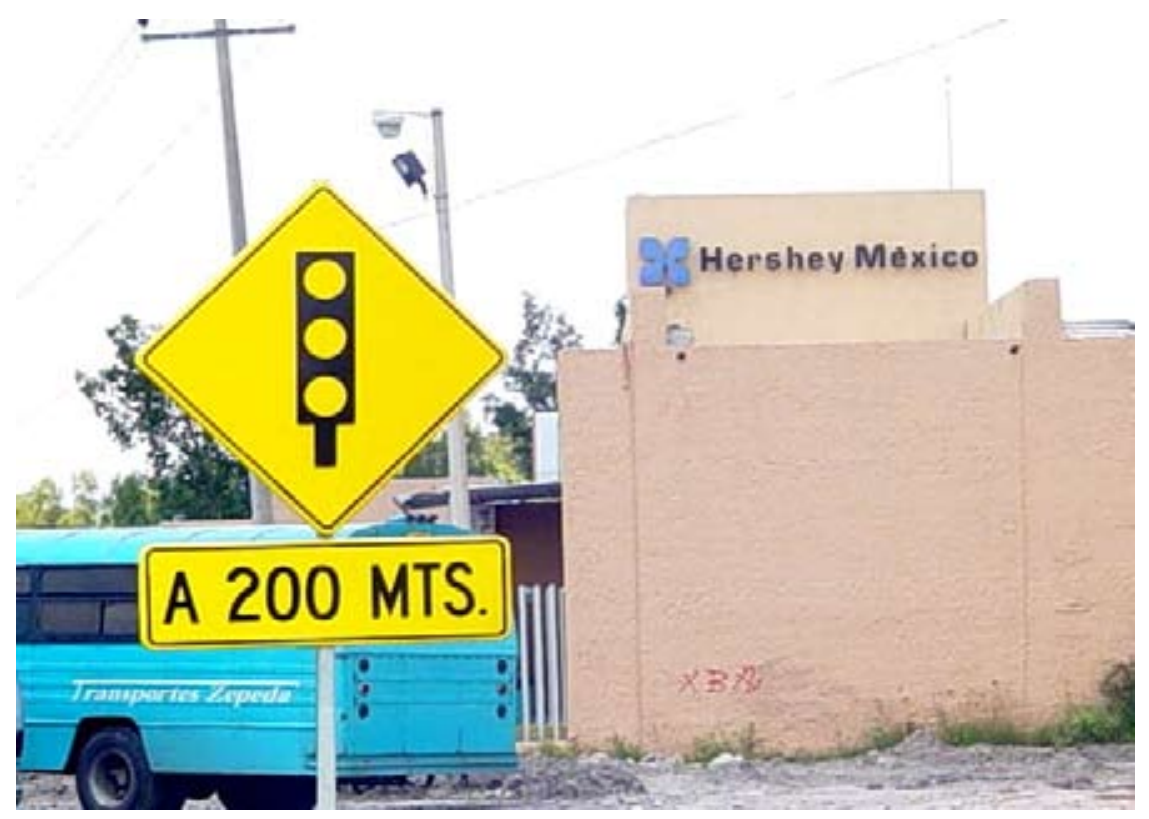

Foto 1 - Existe una fuerte movilidad de la mano de obra hacia el municipio de El Salto FUENTE: Foto Rosario Cota, 2007

La metropolización de Guadalajara inicia en la década de 1940 cuando los municipios de Tlaquepaque y Zapopan se unen funcionalmente con Guadalajara, además, de que la expansión urbana llegó hacia esos municipios. Tonalá fue considerado municipio metropolitano hasta la década de 1970.

El Salto fue catalogado como municipio metropolitano hasta la década de 1980, donde se incorporaron localidades como las Pintas y las Pintitas a la mancha urbana, las cuales tuvieron las tasas de crecimiento más altas (Cuadro 1), sólo superadas por la cabecera municipal de Tonalá. Para la década de 1990. Las Pintitas fue la localidad con mayor crecimiento en la Zona Metropolitana de Guadalajara con una tasa del 13,54\%. 
Cuadro 1 - Población y tasas de crecimiento de las localidades de los municipios metropolitanos, 1980-2000

\begin{tabular}{|c|c|c|c|c|c|c|}
\hline \multirow[b]{2}{*}{ Municipio } & \multirow[b]{2}{*}{$\begin{array}{l}\text { Localidad } \\
\text { censal }\end{array}$} & \multicolumn{3}{|c|}{ Población Censal } & \multicolumn{2}{|c|}{ TCPA } \\
\hline & & 1980 & 1990 & 2000 & $\begin{array}{l}1980- \\
1990\end{array}$ & $\begin{array}{l}1990- \\
2000\end{array}$ \\
\hline & $\begin{array}{c}\text { ZM de } \\
\text { Guadalajara }\end{array}$ & $2,210,143$ & $2,910,515$ & $3,539,706$ & 2.86 & 1.99 \\
\hline 39 & Guadalajara & $1,626,152$ & $1,650,042$ & $1,646,183$ & 0.15 & -0.02 \\
\hline 51 & Juanacatlán & 4,851 & 6,674 & 8,117 & 3.32 & 1.99 \\
\hline 70 & $\begin{array}{l}\text { San José del } \\
\text { castillo }\end{array}$ & 3,025 & 5,979 & 9,768 & 7.22 & 5.07 \\
\hline 70 & El Salto & 9,620 & 11,546 & 18,462 & 1.88 & 4.84 \\
\hline 70 & Las Pintitas & 2,561 & 9,618 & 19,060 & 14.5 & 7.13 \\
\hline 70 & Las Pintas & 835 & 4,446 & 15,681 & 18.66 & 13.54 \\
\hline 70 & $\begin{array}{c}\text { San Jose del } \\
\text { Quince }\end{array}$ & 2,420 & 5,430 & 11,286 & 8.62 & 7.65 \\
\hline 97 & Tlajomulco & 20,860 & 11,567 & 16,177 & -8.85 & 3.44 \\
\hline 97 & $\begin{array}{l}\text { Nicolás R. } \\
\text { Casillas }\end{array}$ & 3,215 & 8,226 & 14,335 & 10.09 & 5.77 \\
\hline 97 & $\begin{array}{c}\text { San Sebastián } \\
\text { el Grande }\end{array}$ & 4,186 & 9,295 & 14,695 & 8.5 & 4.72 \\
\hline 97 & $\begin{array}{c}\text { Santa Cruz } \\
\text { del Valle }\end{array}$ & 2,990 & 4,498 & 13,908 & 4.27 & 12.04 \\
\hline 98 & Tlaquepaque & 135,500 & 328,031 & 458,674 & 9.63 & 3.43 \\
\hline 98 & Santa Anita & 12,371 & 10,172 & 12,842 & -1.98 & 2.38 \\
\hline 101 & Tonalá & 21,407 & 151,190 & 315,278 & 22.14 & 7.68 \\
\hline 120 & Zapopan & 345,390 & 668,323 & 910,690 & 6.99 & 3.17 \\
\hline 120 & $\begin{array}{l}\text { Nuevo } \\
\text { México }\end{array}$ & 8,300 & 11,997 & 26,734 & 3.84 & 8.41 \\
\hline 120 & Tesistán & 8,460 & 13,481 & 27,796 & 4.88 & 7.56 \\
\hline
\end{tabular}

FUENTE: Sobrino, 2003

Esta situación permite sustentar que El municipio de El Salto es uno de los que tienen mayor dinámica y es donde se empieza a localizar la población que busca nuevos espacios de vivienda. Sin embargo, ¿cuál es la función que está teniendo ahora que no ha podido integrarse al nuevo desarrollo industrial? 


\section{La función de El Salto en Guadalajara, bajo las nuevas tendencias Metropolitanas}

Las nuevas tendencias metropolitanas, facilitan dos situaciones: el proceso de reestructuración productiva y las nuevas formas de organización intraurbana. En cada uno de estos dos aspectos la ciudad de Guadalajara se ha mantenido activa, debido a la aparición de la industria electrónica.

La reestructuración productiva nace a raíz de que las empresas trasnacionales del ramo electrónico ven a Guadalajara como un polo importante para desarrollar procesos productivos donde se da un uso intensivo en mano de obra; además existe una experiencia en algunas ramas de este sector, las cuales se establecieron en la ciudad desde la década de 1960; por otro lado la reubicación de la planta IBM en el municipio de El Salto a mediados de la década de 1970 y su cambio en la producción de máquinas de escribir a computadoras, permitió que otras empresas siguieran su ejemplo. Finalmente la generación de una infraestructura favorable para tener mano de obra calificada (universidades y centro educativos tecnológicos), llamaron la atención a decenas de empresas para instalar sus plantas productivas en esta ciudad.

Lo anterior ocasionó que el proceso de industrialización en Guadalajara iniciara con una nueva etapa, la cual consistía en reorganizar su proceso productivo, incorporando mano de obra calificada y automatizando la producción y los procesos administrativos; organizarse en red por medio de sus proveedores para ser más competitivos; delegar funciones a empresas que pudieran hacer mejor las cosas, propiciando el desarrollo de servicios al productor; y por último, reubicarse espacialmente dentro de la ciudad, con la intención de aprovechar la infraestructura generada para el desarrollo industrial.

Esta nueva forma de funcionamiento por parte de las empresas manufactureras en Guadalajara ocasionó un cambio en su estructura: surgen nuevas actividades económicas, dando pie a una alteración de la base económica y la aparición de nuevos espacios económicos en la ciudad, terminando con la hegemonía que mantuvo el centro económico tradicional y dar paso a nuevos espacios industriales, comerciales y de servicio.

La aparición de la rama electrónica (bienes de capital) en la vida industria 
de Guadalajara dió pie para considerar que el sector alimenticio perdiera importancia (Bienes básicos), lo mismo sucedió dentro de los servicios ya que la actividad comercial bajo su participación en el porcentaje de personal ocupado mientras que los servicios productivos incrementaron su porcentaje dando una presencia significativa en la ciudad (Gráfica 1).

Gráfica 1 - Distribución de la PO en los tipos de bienes (85-98)

FUENTE: INEGI, 1985, 1993,1998

Dentro de la organización intraurbana, la dinámica metropolitana vivida en Guadalajara durante los últimos veinte años ha pasado de un proceso de urbanización a uno de desurbanización, ya que el municipio central ha disminuido su tasa de crecimiento hasta ser negativa para la década de 1990. En cambio los municipios periféricos como El Salto y Tlajomulco tienen un crecimiento acelerado (Cuadro 2). Incluso los primeros municipios que se incluyeron en la metrópoli (Tlaquepaque, Zapopan y Tonalá), se encuentran en una etapa de reducción en su tasa de crecimiento. Lo que indica que la expansión metropolitana está alcanzando a territorios cada vez más distantes del municipio central. Esto implica que se empiecen a formar nuevos centros económicos, ya que el vigente no podrá impactar de manera funcional hasta esos lugares. 
Cuadro 2 - Tasas de crecimiento de los municipios metropolitanos de Guadalajara

\begin{tabular}{|l|c|c|c|c|c|c|}
\hline & $\mathbf{5 0 - 6 0}$ & $\mathbf{6 0 - 7 0}$ & $\mathbf{7 0 - 8 0}$ & $\mathbf{8 0 - 9 0}$ & $\mathbf{9 0 - 0 0}$ & $\mathbf{5 0 - 0 0}$ \\
\hline Nacional & 3.07 & 3.28 & 3.32 & 1.97 & 1.83 & 2.69 \\
\hline Zona Metropolitana de Guadalajara & 6.54 & 5.41 & 4.24 & 2.55 & 1.22 & 3.97 \\
\hline Guadalajara & 6.89 & 4.94 & 3.09 & 0.15 & -0.02 & 2.98 \\
\hline Salto, El & 0.84 & 3.21 & 4.86 & 6.77 & 8.21 & 4.75 \\
\hline Tlajomulco de Zuñiga & 3.48 & 2.98 & 3.73 & 3.04 & 6.06 & 3.85 \\
\hline Tlaquepaque & 5.41 & 6.03 & 5.80 & 6.72 & 3.42 & 5.47 \\
\hline Tonala & 3.29 & 4.49 & 7.78 & 12.45 & 7.15 & 6.99 \\
\hline Zapopan & 7.24 & 11.04 & 9.61 & 6.23 & 3.48 & 7.49 \\
\hline
\end{tabular}

FUENTE: Elaboración propia con base en los datos de los Censos de Población en diferentes años

Sobre los nuevos centros económicos de la ciudad, empiezan a desarrollarse bajo la aparición de actividades comerciales e industriales en la periferia de la ciudad. Los centros comerciales que iniciaron en Guadalajara a finales de la década de 1960, atrajeron otras actividades y crearon zonas habitacionales; ahora buscan mercados cautivos como zonas residenciales que pueden facilitar el desarrollo comercial.

Por el lado de las áreas industriales, su planta productiva en muchos de los casos presenta obsolescencia (Foto 2), su atracción es sobre otras actividades, aunque también dinamizan el crecimiento de zonas habitacionales, donde se ubica la población de bajos ingresos, debido al costo del terreno y la incompatibilidad de usos del suelo. El municipio de El Salto es un ejemplo de esta situación, una vez que se empezaron a instalar empresas industriales a lo largo de la carretera a Chapala y poblados contiguos, los terrenos aledaños se fraccionaron, a pesar de que tuvieron problemas de infraestructura urbana, dando lugar a zonas con nivel socioeconómico bajo. 


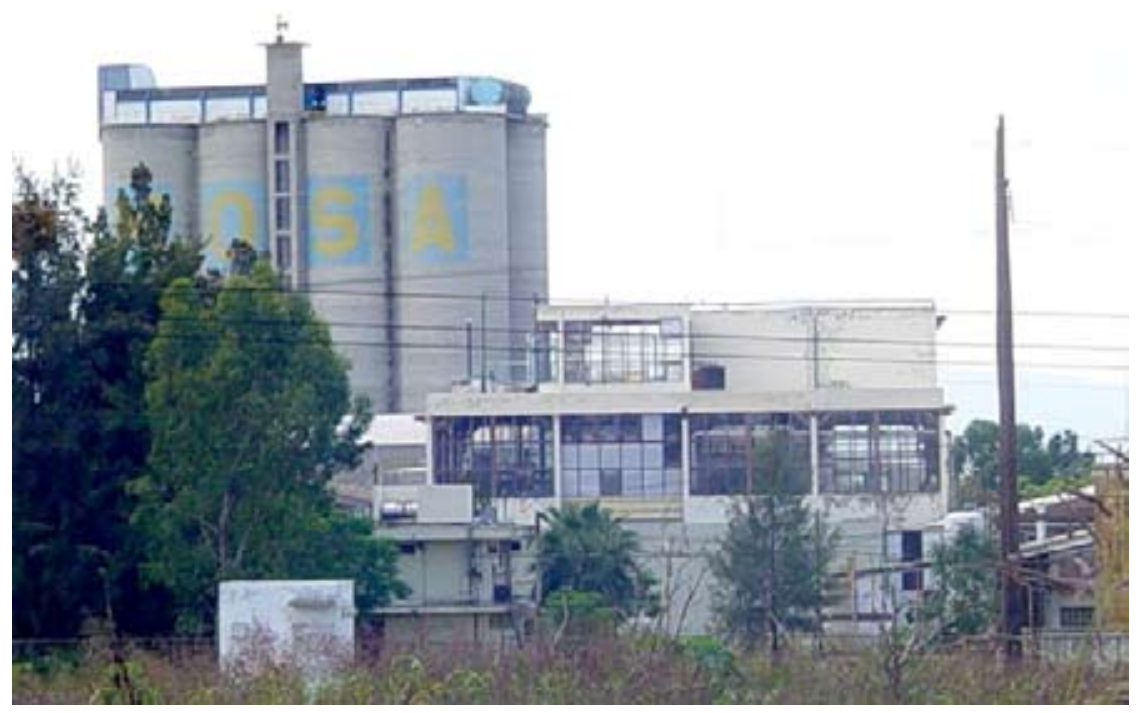

Foto 2 - Obsolescencia de la planta productiva FUENTE: Rosario Cota, 2007

Es así como se define que este municipio tiene la función de sólo recibir a la población que busca suelo barato, donde no existen servicios públicos, infraestructura urbana deficiente, escasez en servicios educativos y de salud. Es decir, en términos generales El Salto es de los municipios que no han aprovechado el desarrollo económico y urbano de la ciudad a partir del proceso de reestructuración productiva. Lo anterior desmotiva la localización de nuevas actividades productivas que permitirían un desarrollo económico local. Una manera de entender la situación que esta viviendo El Salto es comparar los niveles socioeconómicos ${ }^{5}$ que presentan los municipios metropolitanos.

Con base en el censo poblacional del 2000 El Salto ubica el 50\% de

\footnotetext{
${ }^{5}$ Los niveles socioeconómicos son elaborados por el INEGI con una metodología que incluye variables demográficas, de ingreso, educativas, de salud y condiciones de vivienda. El nivel socioeconómico más bajo es el uno lo que implica que sus condiciones de mortalidad, ingreso, nivel educativo, acceso a la salud y dotación de servicios básicos (agua drenaje y electricidad) son muy bajas, en cambio el nivel socioeconómico más alto es el siete lo cual significa que todos sus indicadores son los más altos.
} 
su población en el nivel socioeconómico cuatro, lo que significa que sus condiciones urbanas están limitadas, si se compara con los municipios de Zapopan y Guadalajara, ya que su población se concentra en los niveles siete y seis respectivamente. En cambio los municipios de Tlaquepaque y Tonalá, esta muy similares a El Salto, aunque Tlaquepaque tiene más porcentaje de habitantes en el nivel siete, El Salto es el que mayor porcentaje de habitantes tiene en el nivel tres (Cuadro 3).

Cuadro 3 - Distribución de agebs y población de los niveles socioeconómicos, 2000

\begin{tabular}{|c|c|c|c|c|c|c|c|c|}
\hline \multirow{3}{*}{ Municipios } & \multicolumn{8}{|c|}{ Niveles Socioeconómicos } \\
\hline & \multicolumn{2}{|c|}{7} & \multicolumn{2}{|c|}{6} & \multicolumn{2}{|c|}{4} & \multicolumn{2}{|c|}{3} \\
\hline & agebs & Pob. & agebs & Pob. & agebs & Pob. & agebs & Pob. \\
\hline Guadalajara & 160 & $28.89 \%$ & 265 & $69.21 \%$ & 9 & $1.88 \%$ & 2 & $0.01 \%$ \\
\hline Zapopan & 119 & $38.80 \%$ & 112 & $38.12 \%$ & 111 & $21.82 \%$ & 20 & $1.20 \%$ \\
\hline Tlaquepaque & 14 & $10.35 \%$ & 58 & $42.55 \%$ & 79 & $46.01 \%$ & 14 & $1.07 \%$ \\
\hline Tonalá & 5 & $4.75 \%$ & 41 & $39.98 \%$ & 63 & $50.10 \%$ & 19 & $5.20 \%$ \\
\hline El Salto & 1 & $0.40 \%$ & 22 & $42.35 \%$ & 36 & $50.19 \%$ & 19 & $7.05 \%$ \\
\hline
\end{tabular}

\section{FUENTE: INEGI, Niveles Socioeconómicos, 2000}

$\mathrm{Si}$ se revisan otras condiciones de los municipios para determinar el impacto que está teniendo la actual dinámica industrial y urbana de Guadalajara, es suficiente considerar la distribución de los centros comerciales, las instituciones de educación superior, sobre todo privadas, y los parques industriales.

El caso de los centros comerciales se ubican principalmente en los municipios de Zapopan y Guadalajara, acaparando prácticamente el más del 80 por ciento. Lo anterior se debe por su cercanía con el mercado potencial, pero también por su accesibilidad y su dotación de servicios básicos y urbanos. Esto implica que la concentración esta asociada con las condiciones urbanas de la zona. 
Cuadro 4 - Centros comerciales ubicados en Guadalajara por municipio

\begin{tabular}{|l|c|c|}
\hline Municipio & Número & Porcentaje \\
\hline Zapopan & 20 & 53.0 \\
\hline Guadalajara & 11 & 29.0 \\
\hline Tlaquepaque & 05 & 13.0 \\
\hline Tlajomulco & 01 & 02.5 \\
\hline Tonala & 01 & 02.5 \\
\hline El Salto & 00 & 00.0 \\
\hline Total & 38 & 100.00 \\
\hline
\end{tabular}

FUENTE: INEGI, Censos Económicos, varios años

Por el lado de las instituciones de educación, las públicas tienen una ubicación que dispersa, no tiene que ver con la cercanía del mercado potencial o la infraestructura urbana que se requiere, pero sí las escuelas privadas, las cuales se concentran principalmente en el municipio de Zapopan y sólo algunas instituciones de menor escala se distribuyen en el resto de los municipios metropolitanos.

El caso de los parques industriales como se mencionó anteriormente, estos se concentran en Zapopan, Tlaquepaque y Tlajomulco, aunque existen cuatro en El Salto, estos no han generado un impacto para desarrollar la infraestructura necesaria en ese lugar (Cuadro 5).

Cuadro 5 - Parques industriales ubicados en la zona metropolitana de Guadalajara por municipio

\begin{tabular}{|l|c|c|}
\hline Municipio & Número & Porcentaje \\
\hline Tlaquepaque & 8 & 32 \\
\hline Zapopan & 7 & 27 \\
\hline Tlajomulco & 5 & 18 \\
\hline Guadalajara & 2 & 14 \\
\hline El Salto & 2 & 9 \\
\hline Tonala & 0 & 0 \\
\hline Total & 24 & 100 \\
\hline
\end{tabular}

\section{Reflexiones finales}

La zona metropolitana de Guadalajara es la segunda ciudad más importante de México, después de la capital del país. Su desarrollo industrial y urbano han ido de la mano alcanzado diversas etapas, las cuales han generado un proceso desigual en cada uno de los municipios que conforman 
esta metrópoli.

De los seis municipios El Salto, es uno de los que mantiene las más bajas condiciones de desarrollo urbano. Lo que permite observar las desigualdades que provoca este proceso de desarrollo industrial que esta viviendo la ciudad.

Las políticas locales implementadas en El Salto se han preocupado más por atraer empresas manufactureras, (aunque en los últimos años se observa un fenómeno de retiro de algunas de las empresas manufactureras como es el caso de Hitachi y Euzkadi), que mejorar las condiciones de la población. Esto propicia una mayor desigualdad en el desarrollo urbano de la metrópoli. Aunque la planta productiva en muchos de los casos está sufriendo obsolescencia.

A pesar de que en El Salto se inició un desarrollo industrial, no ha propiciado el bienestar de la población. Además, de que no ha existido un ordenamiento del territorio que permita convivir el desarrollo industrial con el de los habitantes. Lo que implica ser uno de los municipios con menor calidad de vida de la ciudad.

La situación que está viviendo el municipio de El Salto se debe al momento en que se involucró en el proceso de industrialización. Posiblemente los inicios del proceso industrial de este municipio, supuso que seria la zona industrial tradicional de la ciudad.

Durante la etapa del modelo de sustitución de importaciones la participación de El Salto se dio en el momento en que se estaba agotando, a pesar de que se establecieron empresas que serian importantes en la siguiente etapa de industrialización.

Para el desarrollo de la industria electrónica, El Salto no figuró entre los espacios atractivos para la localización de las empresas de este ramo, a pesar de tener áreas industriales. Pero los problemas de accesibilidad, falta de infraestructura y con nulos servicios avanzados, no le quedó otra opción a este municipio más que recibir la población con escasos recursos que buscan suelo barato.

Es así como se puede afirmar que la función que está teniendo este municipio dentro de la zona metropolitana es recibir población segrega de los servicios públicos y sociales, mientras que municipios como Zapopan, Tlaquepaque y Tlajomulco han atraído actividades económicas que permiten dinamizar la economía. 


\section{Referencias Bibliográficas}

ALBA, Carlos; KRUIJT, Dirk. Los empresarios y la industria de Guadalajara. Guadalajara: El Colegio de Jalisco, 1985.

ARIAS, Patricia. La vida económica tapatía durante el siglo XX. In: GARCÍA, Lina Rendón (Coord.). Capitulos de Historia de la ciudad de Guadalajara. Guadalajara: Ayuntamiento de Guadalajara, 1992.

CARAVACA, Inmaculada; MÉNDEZ, Ricardo. Trayectorias industriales metropolitanas: nuevos procesos, nuevos contrastes. Revista EURE, Santiago de Chile, v. 29, n. 87, p. 37-50, ago. 2003.

DE MATTOS, Carlos. Crecimiento metropolitano en América Latina: ¿Los Ángeles como referente? In: International Seminar on Economy and Space. Ouro Preto, Brasil: Centro de Desenvolvimento e Planejamento Regional da Universidade Federal de Minas Gerais, 2001.

DURAND, Jorge. La vida económica tapatía durante el siglo XIX. In: GARCÍA, Lina Rendón (Coord.). Capitulos de Historia de la ciudad de Guadalajara. Guadalajara: Ayuntamiento de Guadalajara, 1992.

EL INFORMADOR. Hemeroteca, Guadalajara, Jalisco.

IMDEC. Instituto Mexicano para el Desarrollo Comunitario AC, 2007.

INEGI. Instituto Nacional de Estadística, Geografía e Informática. X Censo General de Población y Vivienda. México: 1984. . XI Censo General de Población y Vivienda. México: 1990 . XII Censo General de Población y Vivienda. México: 2000. . Censo Económico. México: 1985. . Censo Económico. México: 1988.

. Censo Económico. México: 1993. . Censo Económico. México: 1998. . Niveles Socioeconómicos. México: 2000. 
LECHUGA MONTENEGRO, Jesús. El cambio estructural en la industria manufacturera de Jalisco: hacia un nuevo papel de especialización. Guadalajara: Universidad de Guadalajara, 2002.

MARTÍNEZ REDING, Fernando. Los tapatios, un modo de vivir. Guadalajara: Ayuntamiento de Guadalajara, 1987.

NÚNEEZ MIRANDA, Beatriz. Guadalajara una visión del siglo XX. Guadalajara: Ayuntamiento de Guadalajara, 1999.

PARTIDA, Raquel. Empresas reestructuradas: innovación tecnológica, organización del trabajo y flexibilidad laboral. Guadalajara: CUCSHUDG, 2002.

RIVERA VARGAS, María Isabel. Producción global y desarrollo industrial: de la teoría a la realidad en el Valle del Silicio Mexicano. In: VÁZQUEZ PARADA, Lourdes Celina; O CASTELLANOS, Ana María (Coord.). Cultura y desarrollo regional. Guadalajara: Universidad de Guadalajara, 2002.

RODRÍGUEZ BAUTISTA, Juan Jorge. Procesos de reestructuración económica. El caso de dos ciudades: Monterrey y Guadalajara. Expresión Económica. Guadalajara: Universidad de Guadalajara, 2001.

. La nueva economía y sus efectos en la ciudad de Guadalajara. La última década del siglo XX. Guadalajara: Universidad de Guadalajara, 2006.

SOBRINO, Jaime. Competitividad de las ciudades en México. México: El Colegio de México, 2003.

UNIKEL, Luis. El desarrollo urbano de México: diagnóstico e implicaciones futuras. Con la colaboración de Crescencio Ruiz Chiapeto y Gustavo Garza Villarreal. México: El Colegio de México, 1978. 